\title{
The Era of the Hepatic Oncologist
}

\author{
Ryosuke Tateishi \\ Department of Gastroenterology, Graduate School of Medicine, The University of Tokyo, \\ Tokyo, Japan
}

Traditional phase 1 trials for the development of cytotoxic agents are conducted in order to identify the ideal dose for later phase trials, where the maximum tolerated dose (MTD) is a critical component. The MTD is defined as the prespecified proportion of patients (e.g., 0.2 or 0.33 ) who present dose-limiting toxicity, usually corresponding to grade 3 or higher adverse reactions to the investigational agents (Fig. 1). Generally, the dose is escalated step by step, and a recommended dose for the next phase trial is set 1 step lower than the MTD [1]. This concept is based on the assumption that the higher the dose is the greater the antitumor effect is. It is also assumed that cancer cells are more susceptible to cytotoxic agents than are noncancerous cells due to the significant difference in replication frequency between the two. However, the assumption does not necessarily hold true in the era of molecularly targeted agents (MTA).

Although a dose-response relationship also exists for MTA [2], the relationship is more complicated than that of cytotoxic drugs. For example, gefitinib, an epidermal growth factor receptor (EGFR) tyrosine kinase inhibitor, inhibits cell growth in lung cancer cell lines carrying mutant EGFR in a dose-response fashion. However, this relationship was not observed in cell lines carrying wild-type EGFR [3]. Since the effect of MTA is highly specific to the structure of the target molecule, a small change in the structure results in total ineffectiveness.

The relationship between dosage and toxicity is also more complicated in MTA in which some disabling $\mathrm{AE}$ may occur in later cycles, and a majority of patients experience dose reduction despite not presenting dose-limiting toxicity [4]. This difficulty in dose-ranging studies for MTA resulted in dose modifications in later phase trials. In addition to a larger diversity in the profile and onset period of $\mathrm{AE}$ compared to cytotoxic agents, investigators who engage in the development of MTA for hepatocellular carcinoma (HCC) also have to take into account impaired liver function.

In this issue of Liver Cancer, Maruta et al. [5] explored the efficacy of lenvatinib for advanced HCC patients beyond the inclusion criteria of the phase- 3 clinical trial using real- 


\section{Liver Cancer}

Fig. 1. Traditional concept of the relationship between dose, treatment efficacy, and toxicity in cytotoxic chemotherapy. The solid line indicates the dose-efficacy curve. The dotted line indicates the dose-toxicity curve.

Table 1. Variables considered in the treatment of HCC with molecular targeted agents

\begin{tabular}{|c|c|}
\hline \multicolumn{2}{|l|}{ Liver Cancer 2020;9:378-381 } \\
\hline DOI: 10.1159/000508342 & $\begin{array}{l}\text { (c) } 2020 \text { The Author(s). Published by S. Karger AG, Basel } \\
\text { www.karger.com/lic }\end{array}$ \\
\hline
\end{tabular}

Tateishi: Editorial

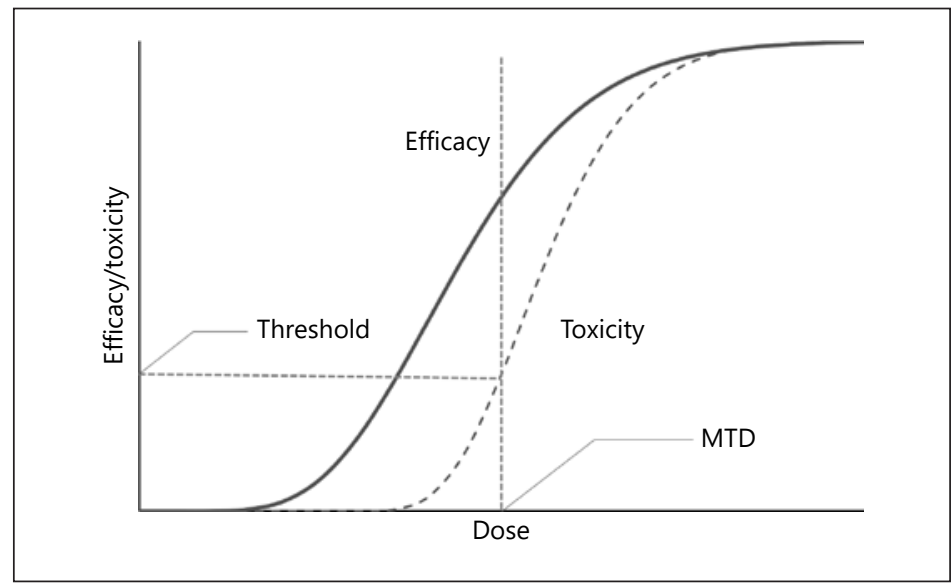

Host-related factors

Body weight/body surface area

Metabolic pathway: hepatic or renal

Individual genetic diversity in drug metabolism

Adaptation: decreased absorption or induced metabolism

Liver function

Tumor-related factors

Sensitivity to the drug

Target cells: cancer cells or vascular endothelial cells

world data. They showed that lenvatinib was valid in a cohort of patients who had main portal vein tumor invasion or in whom lenvatinib was used as a second-line or later treatment. Furthermore, they found that liver-related adverse events including hepatic encephalopathy and bilirubin elevation were more common in patients with Child-Pugh B liver function than in those with Child-Pugh A. In addition, they showed that the 8-week dose intensity of lenvatinib decreased in line with an increase in Child-Pugh score, suggesting that dose modification was required more frequently in those with impaired liver function.

In cytotoxic chemotherapy, the objective response rate increases according to the increased dosage [6], and the actual relative dose intensity (RDI) (i.e., the amount of drug actually administered to each patient during the first several courses) affects overall survival [7]. Recently, several reports have advocated that the RDI is a strong determinant of objective response and a potential predictor of overall survival in the treatment of HCC with lenvatinib [8-11]. Although one has to take into account that the RDI is confounded by other factors including age, performance status, and liver function, as indicated by Maruta et al. [5], these reports suggest that physicians need to make an effort to maintain the RDI to see whether the drug is truly effective and to prolong the duration of the response when the drug is effective.

For that purpose, proper management of adverse events, including not only drug-related ones but also liver-specific complications like hepatic encephalopathy, neutropenia, and thrombocytopenia, is essential. In addition, we should consider various factors, as listed in Table 1, to estimate the actual plasma concentration of MTA. In the phase 3 trial of lenvatinib for unresectable HCC, a body weight-based dosage was adopted based on prior learning from the frequent dose modifications in patients with a lower body weight in the phase 2 trial with a fixed dosage [12]. It is also to be noted that the standard dosage of daily $12 \mathrm{mg}$ of lenvatinib is half of 
that for thyroid cancer, whereas sorafenib, another multikinase inhibitor, adopts the same dosage for HCC, renal cell carcinoma, and thyroid cancer. Both drugs are metabolized mainly by CYP3A4 in the liver $[13,14]$. However, whereas approximately $50 \%$ of sorafenib is excreted as an unchanged form in feces, most of lenvatinib is excreted in feces and urine as metabolites. This suggests that the impact of chronic liver disease is different between the 2 drugs. Since liver function generally deteriorates during treatment in patients with HCC and chronic liver disease, the risk of lenvatinib overdosage may increase according to treatment duration. On the other hand, a decreased serum sorafenib concentration at the time of progression and the need for dose escalation during the treatment course were reported, suggesting decreased absorption from the intestine or autoinduction of sorafenib metabolism [15].

Considering these complicated aspects in MTA-based treatment for advanced HCC, we would conclude that hepatic oncologists who are proficient in dealing with MTA and the management of all kinds of complications related to chronic liver disease, and not general oncologists, should take care of these patients.

\section{Acknowledgement}

We would like to thank Editage (www.editage.com) for English language editing.

\section{Statement of Ethics}

This paper was in accordance with the World Medical Association Declaration of Helsinki. Because this article is an editorial, any type of informed consent is not required.

\section{Disclosure Statement}

Ryosuke Tateishi received honoraria from Eisai and Bayer.

\section{Funding Sources}

None.

\section{Author Contributions}

Conceptualization, writing of this paper, and project administration: Ryosuke Tateishi.

\section{References}

1 Storer BE. Choosing a Phase I Design. In: Crowley J, Hoering A, editors. Handbook of Statistics in Clinical Oncology. 3rd ed. United Kingdom: Chapman and Hall; 2015. pp. 1-19.

2 Gupta S, Hunsberger S, Boerner SA, Rubinstein L, Royds R, Ivy P, et al. Meta-analysis of the relationship between dose and benefit in phase I targeted agent trials. J Natl Cancer Inst. 2012 Dec;104(24):1860-6.

3 Mukohara T, Engelman JA, Hanna NH, Yeap BY, Kobayashi S, Lindeman N, et al. Differential effects of gefitinib and cetuximab on non-small-cell lung cancers bearing epidermal growth factor receptor mutations. J Natl Cancer Inst. 2005 Aug;97(16):1185-94.

4 Postel-Vinay S, Collette L, Paoletti X, Rizzo E, Massard C, Olmos D, et al. Towards new methods for the determination of dose limiting toxicities and the assessment of the recommended dose for further studies of molecularly targeted agents-dose-Limiting Toxicity and Toxicity Assessment Recommendation Group for Early Trials of Targeted therapies, an European Organisation for Research and Treatment of Cancer-led study. Eur J Cancer. 2014 Aug;50(12):2040-9. 
5 Maruta S, Ogasawara S, Ooka Y, Obu M, Inoue M, Itokawa N, et al. Potential of lenvatinib for an expanded indication from the REFLECT trial in patients with advanced hepatocellular carcinoma. Liver Cancer. 2020;1-15.

6 Hryniuk W, Bush H. The importance of dose intensity in chemotherapy of metastatic breast cancer. J Clin Oncol. 1984 Nov;2(11):1281-8.

7 Kwak LW, Halpern J, Olshen RA, Horning SJ. Prognostic significance of actual dose intensity in diffuse large-cell lymphoma: results of a tree-structured survival analysis. J Clin Oncol. 1990 Jun;8(6):963-77.

8 Eso Y, Nakano S, Mishima M, Arasawa S, Iguchi E, Nakamura F, et al. Dose Intensity/Body Surface Area Ratio is a Novel Marker Useful for Predicting Response to Lenvatinib against Hepatocellular Carcinoma. Cancers (Basel). 2019 Dec;12(1):E49.

9 Sasaki R, Fukushima M, Haraguchi M, Miuma S, Miyaaki H, Hidaka M, et al. Response to Lenvatinib Is Associated with Optimal RelativeDose Intensity in Hepatocellular Carcinoma: Experience in Clinical Settings. Cancers (Basel). 2019 Nov;11(11):E1769.

10 Takahashi A, Moriguchi M, Seko Y, Ishikawa H, Yo T, Kimura H, et al. Impact of Relative Dose Intensity of Earlyphase Lenvatinib Treatment on Therapeutic Response in Hepatocellular Carcinoma. Anticancer Res. 2019 Sep;39(9):5149-56.

11 Kuzuya T, Ishigami M, Ito T, Ishizu Y, Honda T, Ishikawa T, et al. Favorable radiological antitumor response at 2 weeks after starting lenvatinib for patients with advanced hepatocellular carcinoma. Hepatol Res. 2020 Mar; 50(3):374-81.

12 Ikeda K, Kudo M, Kawazoe S, Osaki Y, Ikeda M, Okusaka T, et al. Phase 2 study of lenvatinib in patients with advanced hepatocellular carcinoma. J Gastroenterol. 2017 Apr;52(4):512-9.

13 US Food and Drug Administration. Lemvima: highlights of prescribing information. 2018 [cited 2020 Apr 20]. Available from: https://www.accessdata.fda.gov/drugsatfda_docs/label/2018/206947s007lbl.pdf.

14 US Food and Drug Administration. Nexabar: highlights of prescribing information. 2018 [cited 2020 Apr 20]. Available from: https://www.accessdata.fda.gov/drugsatfda_docs/label/2018/021923s020lbl.pdf.

15 Arrondeau J, Mir O, Boudou-Rouquette P, Coriat R, Ropert S, Dumas G, et al. Sorafenib exposure decreases over time in patients with hepatocellular carcinoma. Invest New Drugs. 2012 Oct;30(5):2046-9. 